\title{
Investigating the Motivational and Psychosocial Dynamics of Dysregulated Gaming: Evidence From a Preregistered Cohort Study
}

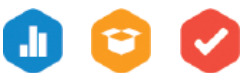

\author{
Andrew K. Przybylski ${ }^{1,2}$ (D) and Netta Weinstein ${ }^{3}$ \\ ${ }^{1}$ Oxford Internet Institute, University of Oxford; ${ }^{2}$ Department of Experimental Psychology, \\ University of Oxford; and ${ }^{3}$ School of Psychology, Cardiff University
}

\begin{abstract}
The American Psychiatric Association (APA) and World Health Organization (WHO) have called for research investigating the clinical relevance of dysregulated video-game play. A growing number of exploratory studies have applied selfdetermination theory to probe the psychological dynamics of problematic gaming, but little is known about these dynamics in adolescents - the targets of most concerns-or the extent to which dysregulated gaming, in turn, affects functioning. In our study of British adolescents and their caregivers $(n=2,008)$, we adopted a confirmatory lens to test the extent to which basic psychological need satisfactions and frustrations underlie dysfunctional gaming behavior. The results, in line with preregistered sampling and data-analysis plans, indicated the frustrations, but not the absence of satisfactions, of psychological needs predicted adolescents' dysregulated gaming and psychosocial functioning. Our discussion focuses on the clinical significance of gaming dysregulation and the advantages of transparent scientific practices for research informed by, and meant to inform, APA and WHO guidance.
\end{abstract}

\section{Keywords}

dysregulated gaming, gaming disorder, Internet gaming disorder, video games, World Health Organization, ICD-11, $D S M-5$, open data, open materials, preregistered

Received 8/21/18; Revision accepted 2/19/19

Video games are now a dominant form of recreation. In the United States, for example, $49 \%$ of adults play games, and $20 \%$ of these adults identify as gamers (Duggan, 2015). This activity is similarly popular in the United Kingdom (46\%), Germany (59\%), France (65\%; Ipsos MORI, 2017), and China (47\%; Ahmad, 2018). The growing popularity of the medium has incited concerns by health-care and mental-health-care professionals that games might lead adolescents and adults to dysregulated, unhealthy patterns of behavior. As a result, the American Psychiatric Associated (APA) and World Health Organization (WHO) proposed that researchers investigate the potential of dysregulated, Internet-based play (APA, 2013; WHO, 2018). These proposals underscore the need for rigorous research to better understand dysfunction associated with dysregulated gaming (van Rooij et al., 2018), which is crucial for determining whether dysregulated gaming merits inclusion as a clinical disorder and the extent to which resources should be allocated toward developing clinical interventions for this form of play.

To date, research on dysregulated gaming has focused primarily on the psychometric properties of questionnaires measuring the phenomenon and its prevalence (Aarseth et al., 2016). Both areas are fraught with debate; for example, prevalence rates vary widely from $46 \%$ in convenience studies (Wan \& Chiou, 2006) to below $1 \%$ of players in population-representative cohorts (Festl, Scharkow, \& Quandt, 2013; Przybylski, Weinstein, \& Murayama, 2016). It has been argued that to reduce such uncertainty and avoid undue burden on

Corresponding Author:

Andrew K. Przybylski, University of Oxford, Oxford Internet Institute, Oxford OX1 3JS, United Kingdom

E-mail: andy.przybylski@oii.ox.ac.uk 
national health systems, investigations of dysregulated gaming should conform to rigorous empirical standards, including open data, materials, and preregistration (Aarseth et al., 2016; van Rooij et al., 2018).

A developing area of research has focused on the motivational underpinnings of dysregulated engagement, applying the motivational framework provided by self-determination theory (SDT; Deci \& Ryan, 2000) to examine how players develop unhealthy passion for gaming (Lafrenière, Vallerand, Donahue, \& Lavigne, 2009; Przybylski, Weinstein, Ryan, \& Rigby, 2009). Results consistent with basic psychological-needs theory (BPNT; Ryan \& Deci, 2017), an SDT microtheory, identify three basic psychological needs as influencing the regulation of gaming: competence, feeling effective in acting on the world and achieving desired outcomes; autonomy, experiencing a sense of choice and psychological freedom; and relatedness, feeling close and connected to others. Notably, it is increasingly clear that along with exploring the satisfaction of these three needs, as has been previously done, experiences of need frustration deserve to be studied in their own right (see also Vansteenkiste \& Ryan, 2013).

Recent APA- and WHO-inspired research on dysregulated gaming has examined relations with psychologicalneed experiences. Focused on online games in line with APA concerns, studies have shown that low levels of psychological-need satisfaction predict more dysregulated gaming (Weinstein, Przybylski, \& Murayama, 2017), that high levels protect against dysregulated gaming (Allen \& Anderson, 2018), and that need frustration presents a risk (Mills, Milyavskaya, Heath, \& Derevensky, 2018). Building on these general trends, findings reported by Allen and colleagues (2018) suggest that the need satisfactions provided by playing games (Przybylski, Rigby, \& Ryan, 2010) could moderate the link between frustrated needs in daily life and dysregulated gaming, such that positive game experiences exacerbate the harmful effects of frustrated needs in daily life by increasing dysregulated gaming. However, the moderation hypothesis did not replicate in a second study (Mills, Milyavskaya, Mettler, \& Heath, 2018). The existing literature suggests that the satisfaction and frustration of psychological needs may be an important reason that a minority of players engage in gaming in a disordered way, but there are several key gaps in our understanding.

First, although the greatest concerns relating to dysregulated gaming have focused on young people (Egli \& Meyers, 1984), no studies to date have examined the motivational dynamics of dysregulated gaming among adolescents. Second, though BPNT has been applied to a number of investigations of gaming, only one has adopted a theory-testing approach (i.e., Weinstein et al., 2017). The other studies were exploratory, relied on convenience samples, and depended on self-reports of motivation, functioning, and gaming provided by participants, making it difficult to know definitively how psychological-need experiences relate to dysregulated play. Third, the extent to which the satisfaction versus frustration of psychological needs drives dysregulated gaming is not clear, though it is possible that both exert influence on dysregulated gaming or that need frustrations play a more dominant role (Chen et al., 2015). Finally, the extent to which dysregulated gaming shapes players' well-being is not yet clear, though this question is key to understanding whether concerns regarding the harmful effects of dysregulated play are warranted. Findings related to short-term (e.g., Przybylski et al., 2009) and general well-being outcomes (e.g., Allen \& Anderson, 2018) suggest that dysregulated play may be harmful, yet other work (Weinstein et al., 2017) shows no direct links. Thus, it is crucial to test the extent to which dysregulated gaming accounts for emotional, peer, behavioral, or conduct problems-all key indicators of functioning in adolescents-over and above variability linked to basic needs. In other words, it is important to know if dysregulated play accounts for incremental variance in daily life psychosocial functioning, given that we might expect in-game basic psychological-needs satisfactions and daily life need frustrations to give rise to problematic patterns of play in the first place.

\section{The Present Research}

The goal of the present research was to clarify the motivational and psychosocial correlates of dysregulated gaming using a rigorous theory-testing approach based on a preregistered sampling and analysis plan. Quantifying dysfunction in these ways is important for determining whether dysregulated gaming should be classified as a clinical disorder and whether resources should be invested in its treatment. To this end, we conducted a cross-sectional study with a large and representative adolescent cohort informed by guidance from the fifth edition of the Diagnostic and Statistical Manual of Mental Disorders (DSM-5; APA, 2013; Kardefelt-Winther, 2014) and self-determination theory (Deci \& Ryan, 2000). We tested six hypotheses concerned with the extent to which adolescents' dysregulated gaming reliably links to basicneed satisfactions and frustrations, need satisfactions experienced within games, and psychosocial functioning as evaluated by adolescents' caregivers.

Hypotheses 1 and 2: We predicted that those high in psychological-need satisfaction would report lower levels of dysregulated gaming, whereas those facing psychological-need frustration would report higher levels of dysregulated gaming. 
Hypothesis 3: We predicted that daily psychologicalneed frustration would relate to more dysregulated gaming insofar as games provided satisfactions which were otherwise lacking in daily life. In other words, we expected that players might be more likely to have an unhealthy relationship with games if games met needs that they felt were frustrated by their experiences in everyday life.

Hypothesis 4: We expected that dysregulated gaming and need frustration would be positively and uniquely related to psychosocial difficulties, whereas need satisfaction would be linked to fewer psychosocial difficulties.

Hypotheses 5 and 6: The final hypotheses focused on the extent to which psychological-need satisfaction and frustration have indirect effects on psychosocial functioning by way of their effects on dysregulated gaming. We hypothesized that dysregulated gaming would mediate the links between psychological-need satisfactions and frustrations and psychosocial functioning reported by parents.

\section{Method}

\section{Participants}

A nationally representative sample of 1,004 adolescents and an equal number of their caregivers living in England, Scotland, and Wales were recruited using geographic data, household socioeconomic class, participant age, and gender factors based on 2011 United Kingdom Census data. The data were collected with the help of the research firm ICM Unlimited using an online survey methodology. The adolescent sample was evenly divided among 14-year-old adolescents $(n=497)$ and 15 -year-old adolescents $(n=507)$. Of these, 540 participants identified as male, 461 as female, and 3 as "other." The sample was predominantly White; $8.1 \%$ of participants reported they were from Black and minority ethnicities. Total combined annual household income mirrored the general population and ranged from $£ 6,500$ (1.9\%) to $£ 150,000$ or more (2.8\%).

\section{Etbical review}

Ethical review for data collection and analysis was conducted by the research ethics committee at the University of Oxford.

\section{Measures}

Adolescents and caregivers completed the following selfreport measures as part of a larger study conducted in
March 2018 to survey the online lives and behaviors of British youth. After providing consent for their own and their children's participation in this web-based survey, caregivers reported on the primary outcome measure: psychosocial functioning of young people. After completing their portion of the study, caregivers were asked to leave the room, and adolescents then completed their portion of the study. Adolescent participants registered their own consent and completed measures of online gaming behavior, as well as measures of dysregulated gaming and daily psychological-need satisfaction and frustration.

\section{Observed variables}

Psychosocial functioning. Caregivers completed the Strengths and Difficulties Questionnaire, a widely used measure of adolescents' social and emotional functioning, which has been validated in community (Goodman, Ford, Simmons, Gatward, \& Meltzer, 2000), academic, and clinical settings (Becker, Hagenberg, Roessner, Woerner, \& Rothenberger, 2004). Caregivers provided answers on the basis of their adolescents' behaviors in the past month using one of three options: "not true" (coded 1), "somewhat true" (coded 2), or "certainly true" (coded 3 ). Their ratings of these 20 items showed high reliability $(\alpha=.89)$, reflecting two facets, including externalizing (i.e., hyperactivity; conduct difficulties; $M=15.48, S D=3.98, \alpha=.81$ ) and internalizing problems (i.e., peer and emotional difficulties; $M=14.75, S D=4.26, \alpha=.85)$.

Gaming behavior. Adolescents reported whether they played games, how much they played, and with whom they played. They were told, "Many young people regularly play video games on computers (e.g. Minecraft), smartphones (e.g. Candy Crush), and gaming consoles (e.g. New Super Mario Bros.)," and were asked, "Do you play video games?" Those who responded in the affirmative were asked, "Please name the games you played most in the past month and please try to be specific. . .." For each of these, adolescents were asked how long they spent playing each on a daily basis and whether they used the Internet to play the game with others. A total of 525 adolescents said they played at least one Internetbased game. Reports of play ranged from less than 30 $\mathrm{min} /$ day to an extreme of 2 participants reporting playing these games for $21 \mathrm{hr} /$ day, though the average was $3 \mathrm{hr}$ $11 \mathrm{~min}(S D=2 \mathrm{hr} 44 \mathrm{~min})$ each day, a figure in line with national statistics (Ipsos MORI, 2017).

Dysregulated gaming. Adolescents who played at least one Internet-based game completed a nine-item checklist drafted in consultation with clinical and research 
psychologists studying video games and behavioral addictions. The checklist was used in previous research to study dysregulated gaming through the lens of the APA's guidance on Internet gaming disorder (IGD; Przybylski et al., 2016; Weinstein et al., 2017). These included items such as "I felt moody or anxious when unable to play," "I felt that I should play less but couldn't," and "I risked friends or opportunities due to games." For those who said they played online games, just under half reported no IGD indicators ( $44 \%, n=233$ ), and the number of participants endorsing dysregulated gaming decreased monotonically from those who reported one symptom $(18.9 \%, n=99)$ to those who reported seven or more $(0.8 \%, n=4)$. Following the analysis plan and approach used in previous research on dysregulated gaming (Przybylski, 2016; Przybylski et al., 2016; Weinstein et al., 2017), responses were summed to create a composite score that ranged from 0 to $9(M=1.33, S D=1.61, \alpha=$ .64). The frequencies of indicator counts are presented in Table S1 in the Supplemental Material available online.

Psychological needs in daily life. Psychological-need satisfaction and frustration were measured using a 24-item self-report scale (Chen et al., 2015). Participants used a 5-point scale to rate the truth of 12 items reflecting need satisfaction in daily life, including "I feel a sense of choice and freedom in the things I undertake" $(M=3.94, S D=0.66$, $\alpha=.92)$ and 12 items reflecting need frustration, including "My daily activities feel like a chain of obligations" ( $M=$ 2.53, $S D=0.95, \alpha=.94)$.

Psychological needs within games. If adolescent participants indicated that they played Internet-based games, they also completed the 12 items measuring psychologicalneed satisfactions using the same general instructions and response anchors used for daily needs, this time in the context of need experiences within games. Participants responded to the same 5-point scale used to measure needs in daily life but focused on games; for example: "When I play online games I feel capable at what I do." Responses were summed to create a composite score for each participant $(M=3.92, S D=0.69, \alpha=.92)$.

\section{Results}

\section{Data and analytic strategy}

We conducted an a priori power analysis to determine the target number of observations needed to provide a fair test of our hypotheses. Because APA and WHO guidance on dysregulated gaming is relatively new and suggests that the impact of dysregulated gaming should be "significant," the literature did not provide an empirical baseline to justify a smallest effect size of interest (SESOI; Lakens, 2017). This left us without a clear anchor to evaluate whether gaming has practically significant impacts on psychosocial outcomes. We used $r^{2}=.04$, the SESOI proposed by Ferguson (2009) for general-media-effects research, though this was modest by traditional standards (Cohen, 1992). We decided that $4.0 \%$ of shared variability between our explanatory and criterion variables was a practically significant effect in line with other gaming outcomes (Hilgard, Engelhardt, \& Rouder, 2017). Power analysis using this SEOSI showed that a sample size of 600 was required for a sensitive test of hypotheses (99\% power). Given that 6 in 10 adolescents play online games (Lenhart, 2015), a target sample of 1,000 adolescents was recruited.

There were four deviations from the analysis plan (Przybylski \& Weinstein, 2018b): (a) The target effect size $\left(r^{2}\right)$ was .039 instead of .04; (b) the effective sample size was smaller than anticipated $(n=525)$; (c) reviewers suggested that we test the first two hypotheses, including covariates, and we agreed; and (d) during analysis, it was clear that dysregulated gaming scores were more skewed and dispersed than expected. We transformed these scores by adding 1 and log-transforming the raw scores for hypothesis testing. We also conducted sensitivity analyses using negative binomial regression models to test if this assumption held and the direction, significance, and magnitude of effects did not change based on the modeling method. These results are available, along with the data, code, and materials, on the Open Science Framework (Przybylski \& Weinstein, 2018a).

\section{Preliminary analyses}

In Table 1, we present the zero-order correlations observed between the variables of interest in this study.

\section{Confirmatory bypothesis testing}

In line with the analysis plan, six hypotheses concerning motivation, psychosocial functioning, and dysregulated gaming were evaluated. The results for Hypotheses 1 through 4 are summarized in Table 2 and those for Hypotheses 5 and 6 are shown in Table S2 in the Supplemental Material.

Hypotbesis 1: psychological-need satisfaction and dysregulated gaming. The first analysis tested the prediction that psychological-need satisfaction is negatively related to dysregulated gaming. In line with our analysis plan, this model did not include covariates. As expected, general-need satisfaction was significantly negatively associated with the criterion, $\beta(523)=-0.155, p=.001$, yet the 
Table 1. Observed Zero-Order Correlations

\begin{tabular}{|c|c|c|c|c|c|c|c|c|}
\hline Variable & 1 & 2 & 3 & 4 & 5 & 6 & 7 & 8 \\
\hline 1. Female & - & & & & & & & \\
\hline 2. Age & .04 & - & & & & & & \\
\hline 3. Psychological-need satisfaction & -.03 & .06 & - & & & & & \\
\hline 4. Psychological-need frustration & .08 & .05 & $-.35^{\text {*** }}$ & - & & & & \\
\hline 5. Externalizing problems & -.01 & .03 & $-.32 * *$ & $.57^{* * *}$ & - & & & \\
\hline 6. Internalizing problems & $.12^{* * *}$ & $.10^{*}$ & $-.28^{* * *}$ & $.65^{* * *}$ & $.62 * *$ & - & & \\
\hline 7. Gaming time & -.08 & .01 & $.10^{*}$ & $.11^{*}$ & .04 & .02 & - & \\
\hline 8. In-game-need satisfaction & -.05 & .05 & $.60^{* * *}$ & $-.09 *$ & $-.13^{* *}$ & -.09 & $.20^{* * *}$ & - \\
\hline 9. Dysregulated gaming & -.08 & .04 & $-.12^{* * *}$ & $.39 * *$ & $.32^{* * *}$ & $.31^{* * *}$ & $.16^{\text {**** }}$ & $.09 *$ \\
\hline
\end{tabular}

${ }^{*} p<.05 .{ }^{* * *} p<.001$.

relation was smaller than predicted, accounting for $2.4 \%$ of the variability in dysregulated gaming. Though the observed effect was significant and in the direction expected, this effect was too small, below the $4.0 \%$ threshold set in the analysis plan, to warrant further study.

Hypotbesis 2: psychological-need frustration and dysregulated gaming. To test the hypothesis that feeling that basic psychological needs frustrated in everyday life may constitute a risk factor for dysregulated gaming, we used a linear model mirroring the one used to test Hypothesis 1. Results indicated that need satisfaction was positively associated with the criterion, $\beta(523)=0.367, p=$ .001. Furthermore, the observed link was not only statistically significant but also accounted for $13 \%$ of the variability in dysregulated gaming, fully supporting the second prediction.

Hypothesis 3: moderating influence of need satisfaction in online games. The third hypothesis concerned the degree to which gaming-need satisfaction moderates the link between psychological-need frustration in day-to-day life and dysregulated gaming. To test this idea, the planned model regressed dysregulated gaming scores onto participant age and gender in the first step (neither were significant, $p s>.08$ ) and onto daily need frustration and gaming-need satisfaction in the second step (both were significant, $p s<.05$ ). Yet the interaction term entered in the third step of the model was itself not significant, $\beta(519)=0.140, p=.61$, such that the third hypothesis was not supported. A related exploratory analysis tested if the relation between psychological-need frustration and game engagement was moderated by in-game need satisfaction, the rationale being that satisfaction of needs during play might provide a distraction from need frustration in daily life.

Hypothesis 4: direct effects of psychosocial functioning. Need satisfaction experienced in daily life was expected to be negatively associated with both externalizing and internalizing problems, whereas the opposite was expected for daily life need frustration and dysregulated gaming, holding variability in participant gender and age constant. The first model regressed externalizing problems simultaneously onto need satisfaction, $\beta(519)=-0.138, p=.001$, need frustration, $\beta(519)=0.474, p<.001$, and dysregulated gaming, $\beta(519)=0.117, p=.003$. Though all three were significantly related to externalizing problems in the directions predicted, only daily need frustration accounted for a practically significant share of variability in this outcome (17\%). The second model examined internalizing problems and showed that need satisfaction, $\beta(519)=$ $-0.067, p=.060$, need frustration, $\beta(519)=0.584, p<.001$, and dysregulated gaming, $\beta(519)=0.075, p=.038$, were related in the direction expected, yet daily need frustration was the only factor that accounted for a practically significant share of variability in internalizing problems (25\%). These results partially supported the fourth hypothesis and highlighted the importance of need frustration.

Hypotheses 5 and 6: indirect effects on psychosocial functioning. Four indirect-effects models evaluated the mediating influence of dysregulated gaming on the relations between daily need satisfaction and frustration and psychosocial functioning (see Fig. S1 and Table S2 in the Supplemental Material). Results broadly mirrored and extended the pattern of findings derived from testing Hypotheses 1, 2, and 4. Need satisfaction and frustration were consistent predictors of both dysregulated gaming (Path $a$ ) and externalizing and internalizing problems (Path $c$ ). Furthermore, all of the hypothesized indirect effects (Path $a \times b$ ) relating needs to psychosocial outcomes by way of dysregulated gaming were statistically significant in the direction hypothesized, yet accounted for little incremental variability $(<0.3 \%)$ when compared with the direct effects of psychological-need satisfaction $(>6 \%)$ and frustration (> 23\%). In other words, daily psychological-need experiences robustly predicted 
Table 2. Results of Regression Models Evaluating Hypotheses 1 to 4

\begin{tabular}{|c|c|c|c|c|c|}
\hline Hypothesis (criterion variable) and explanatory variable & \multicolumn{3}{|c|}{ Slope } & $p$ & $\begin{array}{c}\text { Variance } \\
\text { estimate }\left(R^{2}\right)\end{array}$ \\
\hline \multicolumn{6}{|l|}{ Hypothesis 1 (dysregulated gaming) } \\
\hline \multicolumn{6}{|l|}{ Hypothesis 2 (dysregulated gaming) } \\
\hline Psychological-need frustration & 0.117 & {$[0.093,0.140]$} & 0.398 & .000 & .157 \\
\hline Game-need satisfaction & 0.036 & {$[-0.057,0.129]$} & 0.091 & .444 & .001 \\
\hline Psychological-need Frustration $\times$ Game-Need Satisfaction & 0.005 & {$[-0.030,0.039]$} & 0.075 & .783 & .000 \\
\hline \multicolumn{6}{|l|}{ Hypothesis 4 (externalizing problems) } \\
\hline Dysregulated gaming & 1.673 & {$[0.584,2.761]$} & 0.117 & .003 & .011 \\
\hline Psychological-need satisfaction & -0.827 & {$[-1.274,-0.380]$} & -0.138 & .000 & .017 \\
\hline Psychological-need frustration & 2.633 & {$[2.293,2.973]$} & 0.584 & .000 & .252 \\
\hline
\end{tabular}

Note: $b=$ unstandardized regression coefficient; $C I=$ confidence interval; $\beta=$ standardized regression coefficient.

psychosocial functioning, holding variability relating to gaming and control variables constant, though the opposite was not supported.

\section{Exploratory analyses}

During peer review, it was suggested that psychologicalneed frustration and in-game need satisfaction might interact to predict engagement with online games. Reflecting on SDT and BPNT, we agreed this was an intriguing research question. After controlling for participant gender and age, we found both in-game need satisfaction $(\beta=.21, p<.001)$ and everyday psychologicalneed frustration $(\beta=.13, p=.002)$ were positively associated with the amount of time participants allocated to games, but the moderating term was not significant $(p=.985)$.

\section{Discussion}

The APA and WHO are considering classifying dysregulated gaming as a new clinical diagnosis, but little research has considered the extent to which it represents a dysfunctional form of behavior meriting serious clinical consideration. In the present research, we applied motivational theory to investigate whether psychological-need satisfactions and frustrations in adolescents' daily lives are linked to dysregulated engagement. Our intent was to move beyond the exploratory empirical frames used to study dysregulated gaming by using an explicit theory-testing approach informed by SDT. Results derived from this nationally representative sample confirmed a number of preregistered hypotheses concerning dysregulated online play but did not provide support for others.

The first line of findings demonstrated that daily psychological-need frustration, in particular, consistently related to dysregulated gaming. These results are in line with previous ones (Mills, Milyavskaya, Heath, et al., 2018), yet the amount of variance accounted for was less than the criterion we set for a clinically significant effect. We did not find evidence that low levels of need satisfaction predicted variability in dysregulated gaming over and above psychological-need frustration. This latter finding makes sense, given that having one's needs frustrated is more likely to account for dysregulation, and mirrors findings in analogue domains, including education (Chen et al., 2015), sports (Bartholomew, Ntoumanis, Ryan, Bosch, \& Thogersen-Ntoumani, 2011), and in relation to other dysregulated behaviors such as disordered eating (Boone, Vansteenkiste, Soenens, Van der KaapDeeder, \& Verstuyf, 2014). In addition, results from testing our moderation hypothesis inform conflicting results in the existing literature (Mills, Milyavskaya, Mettler, et al., 2018); we did not find evidence that psychologicalneed satisfaction experienced within games moderates the link between basic-need frustration and dysregulated gaming. This null finding runs counter to previously reported moderation effects on dysregulated gaming (Allen \& Anderson, 2018), suggesting that future research should explore which factors of specific games or samples of gamers might result in divergent findings. This being understood, it is clear that the explanatory and predictive power of BPNT with respect to dysregulated 
gaming is rooted in psychological-need frustration. Though basic psychological-need satisfactions provided in everyday life and in games might be correlated with gaming engagement more broadly (Przybylski et al., 2010), neither accounts for significant variability in dysregulated gaming when basic-need frustration is considered.

The second set of the findings concerning adolescents' psychosocial functioning were noteworthy. In line with what would be expected by BPNT, daily psychological-need satisfaction and need frustration were associated with both externalizing and internalizing problems (Ryan \& Deci, 2017). Furthermore, whereas dysregulated gaming was negatively correlated with psychosocial functioning, as expected, it accounted for a practically insignificant share of variability in key outcomes, a very small effect as compared with the role played by basic psychological needs. Given that many have argued that the clinical value of dysregulated gaming is determined in large part by its impact on functioning, our findings are noteworthy (WHO, 2018) and strongly suggest that dysregulated gaming is unlikely to be a practically significant route by which psychologicalneed frustration undermines psychosocial functioning. Supporting this conclusion, results derived from testing Hypotheses 5 and 6 indicated that there are measurable indirect effects linking psychological needs to psychosocial functioning by way of dysregulated gaming, but these relations were considerably smaller than we expected. This evidence suggests that having information about the extent to which an adolescent's videogame play is dysregulated provides no practically useful incremental information when viewed in light caregivers' assessments of emotional, behavioral, peer, or conduct difficulties.

\section{Limitations and methodological considerations}

This research presents three noteworthy limitations. First, data were strictly correlational. On the basis of extant theory and evidence (Weinstein et al., 2017), we have some confidence that basic psychological-need experiences reliably precede dysregulation (Chen et al., 2015), but further research is needed to probe this. Such work should be conducted with sensitive hypothesis testing, as future studies examining the interrelation between daily need frustration and dysregulated gaming and health should be understood as a medium effect $(r \approx 36)$, whereas those wanting to reliably study dysregulated gaming effects $(r \approx .07)$ will need to recruit much larger samples if we assume that the betweenperson effects observed here are mirrored in terms of within-person trends over time. Those studying dysregulated gaming will also need to develop new methods for dealing with data distributions with high dispersion.

Third, findings were interpreted using a widely cited recommendation (Ferguson, 2009) for our SESOI. This is in line with a clinical-outcomes literature that suggests that those studying the impact of dysregulated gaming on clinical outcomes should adopt a more stringent SESOI so that the effects of technology on clinical outcomes constitute a minimally important difference (MID; Miller, 1956; Norman, Sloan, \& Wyrwich, 2003). Although the MID is more conservative than our SESOI $\left(r^{2}=.059\right.$ vs. .04), adopting it would not have affected our findings: Gaming effects fell well below and need frustration were well above the MID. However, researchers interpreting findings in light of MID recommendations could collect a smaller and less-expensive sample without sacrificing statistical power $(n \approx 296 ; 1-\beta=$ $0.99)$. Given that our large sample size meant that interpretations of data varied widely when using statistical significance versus a SESOI, we argue that those studying dysregulated gaming should adopt a SESOI as standard in future research, particularly when recruiting large samples. Doing so will provide an informed basis for power analysis and will minimize the chance that researches will interpret statistically, but not practically significant gaming effects as clinically relevant.

Finally, our inferences are based on a sample drawn from the general population of adolescents living in Great Britain. We did not collect multinational data, as has been previously done (Przybylski et al., 2016), and we did not focus on a clinical population or use an in-person clinical assessment to measure psychosocial functioning (Kardefelt-Winther, 2014; Van Rooij \& Kardefelt-Winther, 2017). As this research area matures and we move toward building an evidence base suitable for studies leading toward the next revision of the Diagnostic and Statistical Manual of Mental Disorders and International Classification of Diseases, incorporating all three of these will be needed.

\section{Closing remarks}

Health organizations require robust evidence to inform their ongoing decision making regarding whether dysregulated gaming constitutes a significant psychiatric condition meriting clinical attention and resources. Given the high professional and reputational stakes, research meeting this need must be of the highest empirical and theoretical standards. The present work represents a concerted effort to investigate the phenomenon as outlined by the APA and WHO; we complemented rich theory with transparent scientific practices. The findings underscore the importance of experiences of need frustration as a robust predictor of 
both dysregulated gaming and psychosocial functioning. Our approach provides a template for researchers to expand on the scope of findings from this study; this program of work is needed to determine if the attention that researchers and clinicians give this immensely popular activity is empirically justified. Judged on the basis of the evidence reported in this study, we would conclude it is not. In this case, the negative results are highly significant for clinical researchers and health policymakers at the APA and WHO.

\section{Action Editor}

Kenneth J. Sher served as action editor for this article.

\section{Author Contributions}

A. K. Przybylski and N. Weinstein conceptualized the project. A. K. Przybylski conducted data analyses. Both authors take full responsibility for the integrity and accuracy of the data analysis. Both authors approved the final version for submission.

\section{ORCID iD}

Andrew K. Przybylski (iD https://orcid.org/0000-0001-5547-2185

\section{Acknowledgments}

The content of this article is drawn from a larger project but data presented here are not reported elsewhere.

\section{Declaration of Conflicting Interests}

The author(s) declared that there were no conflicts of interest with respect to the authorship or the publication of this article.

\section{Funding}

This research was partially funded by John Fell Fund Grant 163/079 through the University of Oxford and from the British Academy/Leverhulme Trust Small Research Grant SG171197. A. K. Przybylski is supported by grants from Barnardo's UK and an Understanding Society Policy Fellowship Grant ES/ K005146/1. These organizations did not play any role in the study design, analysis, or presentation of these data, and they were not involved in the decision to write this article.

\section{Supplemental Material}

Additional supporting information can be found at http:// journals.sagepub.com/doi/suppl/10.1177/2167702619859341

\section{Open Practices

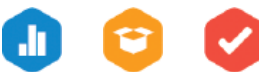

All data and materials have been made publicly available via Open Science Framework and can be accessed at https://osf .io/5fbac/ and https://osf.io/gx4j7/, respectively. The design and analysis plans for the experiments were preregistered at https:// osf.io/gx4j7/. The complete Open Practices Disclosure for this article can be found at http://journals.sagepub.com/doi/suppl/ $10.1177 / 2167702619859341$. This article has received badges for Open Data, Open Materials, and Preregistration. More information about the Open Practices badges can be found at https://www .psychologicalscience.org/publications/badges.

\section{References}

Aarseth, E., Bean, A. M., Boonen, H., Colder Carras, M., Coulson, M., Das, D., . . . Van Rooij, A. J. (2016). Scholars' open debate paper on the World Health Organization ICD-11 gaming disorder proposal. Journal of Behavioral Addictions, 1, 267-270.

Ahmad, D. (2018). China games market model. Retrieved from http://nikopartners.com/china-games-market-model/

Allen, J. J., \& Anderson, C. A. (2018). Satisfaction and frustration of basic psychological needs in the real world and in video games predict Internet gaming disorder scores and well-being. Computers in Human Behavior, 84, 220-229.

American Psychiatric Association. (2013). Diagnostic and statistical manual of mental health disorders (5th ed.). Washington, DC: Author.

Bartholomew, K. J., Ntoumanis, N., Ryan, R. M., Bosch, J. A., \& Thogersen-Ntoumani, C. (2011). Self-determination theory and diminished functioning: The role of interpersonal control and psychological need thwarting. Personality and Social Psychology Bulletin, 37, 1459-1473.

Becker, A., Hagenberg, N., Roessner, V., Woerner, W., \& Rothenberger, A. (2004). Evaluation of the self-reported SDQ in a clinical setting: Do self-reports tell us more than ratings by adult informants? European Child \& Adolescent Psychiatry, 13(Suppl. 2), II17-II24.

Boone, L., Vansteenkiste, M., Soenens, B., Van der KaapDeeder, J., \& Verstuyf, J. (2014). Self-critical perfectionism and binge eating symptoms: A longitudinal test of the intervening role of psychological need frustration. Journal of Counseling Psychology, 61, 363-373.

Chen, B., Vansteenkiste, M., Beyers, W., Boone, L., Deci, E. L., Van der Kaap-Deeder, J., . . . Verstuyf, J. (2015). Basic psychological need satisfaction, need frustration, and need strength across four cultures. Motivation and Emotion, 39, 216-236.

Cohen, J. (1992). A power primer. Psychological Bulletin, 112, 155-159.

Deci, E. L., \& Ryan, R. M. (2000). The "what" and "why" of goal pursuits: Human needs and the self-determination of behavior. Psychological Inquiry, 11, 227-268.

Duggan, M. (2015, December 15). Gaming and gamers Retrieved from http://www.pewinternet.org/2015/12/15/ gaming-and-gamers/

Egli, E. A., \& Meyers, L. S. (1984). The role of video game playing in adolescent life: Is there reason to be concerned? Bulletin of the Psychonomic Society, 22, 309-312.

Ferguson, C. J. (2009). An effect size primer: A guide for clinicians and researchers. Professional Psychology: Research and Practice, 40, 532-538. 
Festl, R., Scharkow, M., \& Quandt, T. (2013). Problematic computer game use among adolescents, younger and older adults. Addiction, 108, 592-599.

Goodman, R., Ford, T., Simmons, H., Gatward, R., \& Meltzer, H. (2000). Using the Strengths and Difficulties Questionnaire (SDQ) to screen for child psychiatric disorders in a community sample. The British Journal of Psychiatry: The Journal of Mental Science, 177, 534-539.

Hilgard, J., Engelhardt, C. R., \& Rouder, J. N. (2017). Overstated evidence for short-term effects of violent games on affect and behavior: A reanalysis of Anderson et al. (2010). Psychological Bulletin, 143, 757-774.

Ipsos MORI. (2017). Interactive Software Federation of Europe GameTrack digest. Retrieved from https://www.isfe.eu/ wp-content/uploads/2019/01/gametrack_european_summary_ data_2017_q4.pdf

Kardefelt-Winther, D. (2014). Meeting the unique challenges of assessing Internet gaming disorder. Addiction, 109, 1568-1570.

Lafrenière, M.-A. K., Vallerand, R. J., Donahue, E. G., \& Lavigne, G. L. (2009). On the costs and benefits of gaming: The role of passion. CyberPsychology \& Behavior, 12, 285-290.

Lakens, D. (2017 May 11). How a power analysis implicitly reveals the smallest effect size you care about [Blog post]. Retrieved from http://daniellakens.blogspot .com/2017/05/how-power-analysis-implicitly-reveals.html

Lenhart. (2015, April 9). Teens, social media \& technology overview 2015. Retrieved from http://www.pewinternet .org/2015/04/09/teens-social-media-technology-2015/

Miller, G. A. (1956). The magical number seven, plus or minus two: Some limits on our capacity for processing information. Psychological Review, 63, 81-97.

Mills, D. J., Milyavskaya, M., Heath, N. L., \& Derevensky, J. L. (2018). Gaming motivation and problematic video gaming: The role of needs frustration: Motivation, need frustration, problem gaming. European Journal of Social Psychology, 48, 551-559.

Mills, D. J., Milyavskaya, M., Mettler, J., \& Heath, N. L. (2018). Exploring the pull and push underlying problem video game use: A self-determination theory approach. Personality and Individual Differences, 135, 176-181.

Norman, G. R., Sloan, J. A., \& Wyrwich, K. W. (2003). Interpretation of changes in health-related quality of life: The remarkable universality of half a standard deviation. Medical Care, 41, 582-592.
Przybylski, A. K. (2016). Mischievous responding in Internet gaming disorder research. PeerJ, 4, Article e2401. doi:10.7717/ peerj. 2401

Przybylski, A. K., Rigby, C. S., \& Ryan, R. M. (2010). A motivational model of video game engagement. Review of General Psychology, 14, 154-166.

Przybylski, A. K., \& Weinstein, N. (2018a). Motivational and psychosocial correlates of Internet gaming disorder [Data file and code book]. doi:10.17605/osf.io/mqv5k

Przybylski, A. K., \& Weinstein, N. (2018b). Study preregistration. Retrieved from https://osf.io/gx4j7/

Przybylski, A. K., Weinstein, N., \& Murayama, K. (2016). Internet gaming disorder: Investigating the clinical relevance of a new phenomenon. American Journal of Psychiatry. doi:10.1176/appi.ajp.2016.16020224

Przybylski, A. K., Weinstein, N., Ryan, R. M., \& Rigby, C. S. (2009). Having to versus wanting to play: Background and consequences of harmonious versus obsessive engagement in video games. CyberPsychology \& Behavior, 12, 485-492.

Ryan, R. M., \& Deci, E. L. (2017). Self-determination theory: Basic psychological needs in motivation, development, and wellness. New York, NY: Guilford Press.

van Rooij, A. J., Ferguson, C. J., Colder Carras, M., KardefeltWinther, D., Shi, J., Aarseth, E., . . Przybylski, A. K. (2018). A weak scientific basis for gaming disorder: Let us err on the side of caution. Journal of Behavioral Addictions, 7, $1-9$.

Van Rooij, A. J., \& Kardefelt-Winther, D. (2017). Lost in the chaos: Flawed literature should not generate new disorders. Journal of Behavioral Addictions, 6, 128-132.

Vansteenkiste, M., \& Ryan, R. M. (2013). On psychological growth and vulnerability: Basic psychological need satisfaction and need frustration as a unifying principle. Journal of Psychotherapy Integration, 23, 263-280. doi:10.1037/a0032359

Wan, C.-S., \& Chiou, W.-B. (2006). Psychological motives and online games addiction: A test of flow theory and humanistic needs theory for Taiwanese adolescents. CyberPsychology \& Behavior, 9, 317-324.

Weinstein, N., Przybylski, A. K., \& Murayama, K. (2017). A prospective study of the motivational and health dynamics of Internet gaming disorder. PeerJ, 5, Article e3838. doi: $10.7717 /$ peerj. 3838

World Health Organization. (2018). Gaming disorder. Retrieved from http://www.who.int/features/qa/gamingdisorder/en/ 